\title{
A lepto-hadronic model for the multiwavelength emission from the jets of radiogalaxies
}

\section{C. Medina}

IRFU, Service de Physique de Particules, CEA, Saclay, France

E-mail: clementina.medina@cea.fn

\section{M. Reynoso}

INFIMAR (UNMDP - CONICET), Mar del Plata, Argentina

E-mail: mreynoso@mdp.edu.ar

\section{G. E. Romero}

Instituto Argentino de Radioastronomía (CCT La Plata-CONICET), Villa Elisa, Argentina

E-mail: romerodfcaglp.unlp.edu.ar

\begin{abstract}
We present a lepto-hadronic model for the VHE emission from the relativistic jets of FR I radiogalaxies. We assume that protons and electrons are accelerated in a compact region near the base of the jet, and they cool emitting multiwavelength radiation as they propagate along the jet. The particle distributions are obtained using an inhomogeneous steady-state transport equation that accounts for the cooling processes as well as the convection of particles in the jet. The dominant processes that contribute to the photon SED are electron and proton synchrotron radiation, inverse Compton interactions, and the inelastic collisions $p p$ and $p \gamma$. The cases of Cen A and M 87 are studied. The accompanying neutrino output is obtained and the possibility of detection with $\mathrm{Km} 3 \mathrm{Net}$ and IceCube is also discussed.
\end{abstract}

25th Texas Symposium on Relativistic Astrophysics - TEXAS 2010

December 06-10, 2010

Heidelberg, Germany 


\section{Introduction}

Several theoretical models have been proposed to explain the electromagnetic emission of the AGNs. It is commonly accepted that the high-energy radiation is emitted by particles accelerated in the relativistic jets launched from the inner parts of an accretion disk surrounding the central black hole. In general, the high-energy spectral energy distribution (SED) of AGNs presents two characteristic bumps. The lower energy bump, located at optical to X-ray energies, is usually explained as synchrotron emission of electrons while the origin of the high-energy peak in the SED is still under debate (see e.g. [1] for a review). Leptonic models assign this component to inverse-Compton upscattering of synchrotron or external photons from the disk and/or radiation reprocessed in nearby clouds (see e.g [2, 3, 那). In hadronic models, interactions of highly relativistic protons in the jet with ambient matter and photon fields, proton-induced cascades, or synchrotron radiation of protons, are responsible for the high-energy photons (see e.g. [5, 6, 7, 8]). There also exist models which are not based on the emission of accelerated particles in the relativistic jet and suppose the production of $\mathrm{TeV} \gamma$-rays in a pulsar-like cascade mechanism in the magnetosphere of the black hole [9, 10].

The recently reported detection by HESS of the nearby radiogalaxy Cen A [11] is of great relevance as it establishes radiogalaxies as VHE $\gamma$-ray emitters. Cen A is the second non-blazar AGN discovered at VHE, after the HEGRA detection of $\gamma$-rays from M 87 [12] and the posterior confirmation by HESS [13].

During the first year of operation, Fermi/LAT has detected HE emission from Centaurus A [14] and M 87 [15], providing new constraints to the models.

In this work we present a lepto-hadronic model for the emission from FR I radiogalaxies. In Section 2 we present the outline of our scenario, describing its most relevant characteristics. In Section 3 we present the application to Centaurus A and M87. Finally, in Section $\bigoplus$ we discuss on the model implications and perspectives.

\section{Model description}

We assume that a population of relativistic particles can be accelerated to very high energies close to the base of the AGN jet. These primary electrons and protons carry a fraction of the total kinetic power of the jet $L_{\mathrm{j}}^{(\mathrm{kin})}$, and as they are dragged along with the jet, they cool giving rise to electromagnetic emission and neutrinos. Most of the jet content is in the form of a thermal plasma with a constant bulk Lorentz factor $\Gamma_{\mathrm{b}}$. This plasma is initially in equipartition with a tangled magnetic field at the Alfvén surface (see e.g. [16]). The jet is accelerated through the conversion of magnetic energy into kinetic energy (e.g. [17]). We adopt a phenomenological dependence on the distance to the black hole for the magnetic field [18] given by $B(z)=B_{0}\left(\frac{z_{0}}{z}\right)^{m}$ with $m \in(1,2)$.

The particle acceleration takes place in a compact region of size $\Delta z<z_{\text {acc }}$ near the base of the jet, at a distance $z_{\text {acc }}$ away from the black hole. The value of $z_{\text {acc }}$ is fixed by requiring the magnetic energy density to be in sub-partition with the jet kinetic energy density.

The power injected in the form of relativistic particles $\left(L_{\mathrm{rel}}\right)$ is considered to be a fraction $q_{\text {rel }}$ of the total jet kinetic power and the relation between proton and electron powers is given by the parameter $a$ such that $L_{p}=a L_{e}$. The energy distribution of the different particle populations 
along the jet is obtained in the jet frame, where the distributions are isotropic, as solutions of a 1dimessional steady state transport equation that includes the relevant cooling terms and a convective one:

$$
v_{\text {conv }}(z) \frac{\partial N(E, z)}{\partial z}+\frac{\partial[b(E, z) N(E, z)]}{\partial E}+\frac{N(E, z)}{T_{\mathrm{d}}(E)}=Q(E, z),
$$

where the effects of convection are taken into account on the term with the constant speed $v_{\text {conv }}$ (close to the jet velocity) and particle cooling with an energy loss $b(E, z)=-d E / d t$. Particle decay with a timescale $T_{\mathrm{d}}(E)$ is also considered in the cases of secondary pions and muons. The source term is given by a function $Q(E, z)$, which in the case of primary particles is given by (in the jet frame)

$$
Q_{i}(E, z)=K_{i}\left(\frac{z_{\mathrm{acc}}}{z}\right)^{2} E^{-s} \exp \left[-\left(\frac{E}{E_{i}^{(\max )}}\right)\right]\left[\mathrm{GeV}^{-1} \mathrm{~s}^{-1} \mathrm{~cm}^{-3} \mathrm{sr}^{-1}\right] .
$$

For secondaries it is obtained using the parent particle distribution $(N(E, z))$ along with the secondary particle production rate.

The maximun energies are obtained from the balance of the main energy loses and gains considering that particles are accelerated by shocks. The main cooling process considered for electrons is synchrotron. These photons are the target for Inverse Compton up-scattering and $p \gamma$ interactions. They also cause internal absorption of $\gamma$-rays. In the case of protons we take into account also the adiabatic expansion, $p p$ and $p \gamma$ interactions.

The radiative output is obtained in the jet reference frame and the result is transformed back to the observer frame. The main radiative processes considered in this work are: synchrotron radiation, IC emission, $p p$ and $p \gamma$ interactions.

We finally consider the absorption of the emitted radiation due to photoionization processes at $\mathrm{eV}$ energies, and also due to $e^{-} e^{+}$creation by $\gamma \gamma$ and $\gamma N$ interactions at high energies in the source. For $\gamma N$ interactions, we take as target the material along the line of sight corresponding to each particular object, which is measured through the column density of neutral hydrogen $N_{H}$. The absorption cross section $\sigma_{\gamma H}$ in this case is taken as in [19] for $E_{\gamma}<1 \mathrm{keV}$ assuming that the medium is composed mainly by atomic hydrogen and dust within galactic abundances. This cross section includes the effects of photoionizaton for $E_{\gamma}>13.6 \mathrm{eV}$ and scattering with dust below this energy. For energies above $1 \mathrm{keV}$ we take the $\gamma N$ cross section from [20], to account for Compton scattering and $e^{ \pm}$production. The resulting optical depth for $\gamma H$ interactions is approximated as $\tau_{\gamma H}\left(E_{\gamma}\right)=N_{H} \sigma_{\gamma H}\left(E_{\gamma}\right)$. In the case of $\gamma \gamma$ interactions, the main radiation target is considered to be the synchrotron photons inside the jet. If a dissipationless accretion disk is present, no other significant photon field is relevant for $\gamma$-ray absorption.

\section{Application to Cen A and M87}

In this section we present the results for the case of Centaurus A and M87 which were obtained applying our model with the set of parameters listed on Table 1. The cooling rates for high energy electrons and protons for these configurations are also shown in Fig. 1.

We can see in Fig. 1 1 that the relevant energy looses are due to synchrotron cooling for electrons and $p \gamma$ interactions for protons. Only at $z=z_{\text {acc }}, p \gamma$ interactions are favored by a large density of 
Table 1: Model parameters for Cen A and M87

\begin{tabular}{lll}
\hline Parameter & CenA & M87 \\
\hline$M_{\mathrm{bh}}:$ black hole mass & $10^{8} M_{\odot}$ & $6 \times 10^{9} M_{\odot}$ \\
$R_{\mathrm{g}}:$ gravitational radius & $1.47 \times 10^{13} \mathrm{~cm}$ & $8.87 \times 10^{14} \mathrm{~cm}$ \\
$L_{\mathrm{j}}^{(\mathrm{kin})}:$ jet kinetic power at $z_{0}$ & $6.28 \times 10^{44} \mathrm{erg} \mathrm{s}^{-1}$ & $1.8 \times 10^{46} \mathrm{erg} \mathrm{s}^{-1}$ \\
$q_{\mathrm{j}}:$ ratio $2 L_{\mathrm{j}}^{(\text {kin })} / L_{\mathrm{Edd}}$ & 0.1 & 0.1 \\
$\Gamma_{\mathrm{b}}\left(z_{0}\right):$ bulk Lorentz factor of the jet at $z_{0}$ & 3 & 4 \\
$\theta:$ viewing angle & $30^{\circ}$ & $30^{\circ}$ \\
$\xi_{\mathrm{j}}:$ jet's half-opening angle & $2.5^{\circ}$ & $1.5^{\circ}$ \\
$q_{\mathrm{rel}}:$ jet's content of relativistic particles & 0.05 & 0.1 \\
$a:$ hadron-to-lepton power ratio & 0.02 & 40 \\
$z_{0}:$ jet's launching point & $50 R_{\mathrm{g}}$ & $50 R_{\mathrm{g}}$ \\
$z_{\mathrm{acc}}:$ injection point & $131 R_{\mathrm{g}}$ & $166 R_{\mathrm{g}}$ \\
$B\left(z_{\text {acc }}\right):$ magnetic field at $z_{\text {acc }}$ & $3065 \mathrm{G}$ & $502.8 \mathrm{G}$ \\
$s:$ spectral index injection & 1.8 & 2.4 \\
$\eta:$ acceleration efficiency & $1.0 \times 10^{-2}$ & $10^{-4}$ \\
$E_{p}^{(\text {min })}:$ minimum proton energy & $3.3 \mathrm{GeV}$ & $2.90 \mathrm{GeV}$ \\
$E_{e}^{(\text {min })}:$ minimum electron energy & $7.0 \times 10^{-2} \mathrm{GeV}$ & $6.0 \times 10^{-2} \mathrm{GeV}$ \\
$N_{H}:$ column dust density & $10^{23} \mathrm{~cm}^{-2}$ & $2 \times 10^{20} \mathrm{~cm}^{-2}$ \\
\hline
\end{tabular}
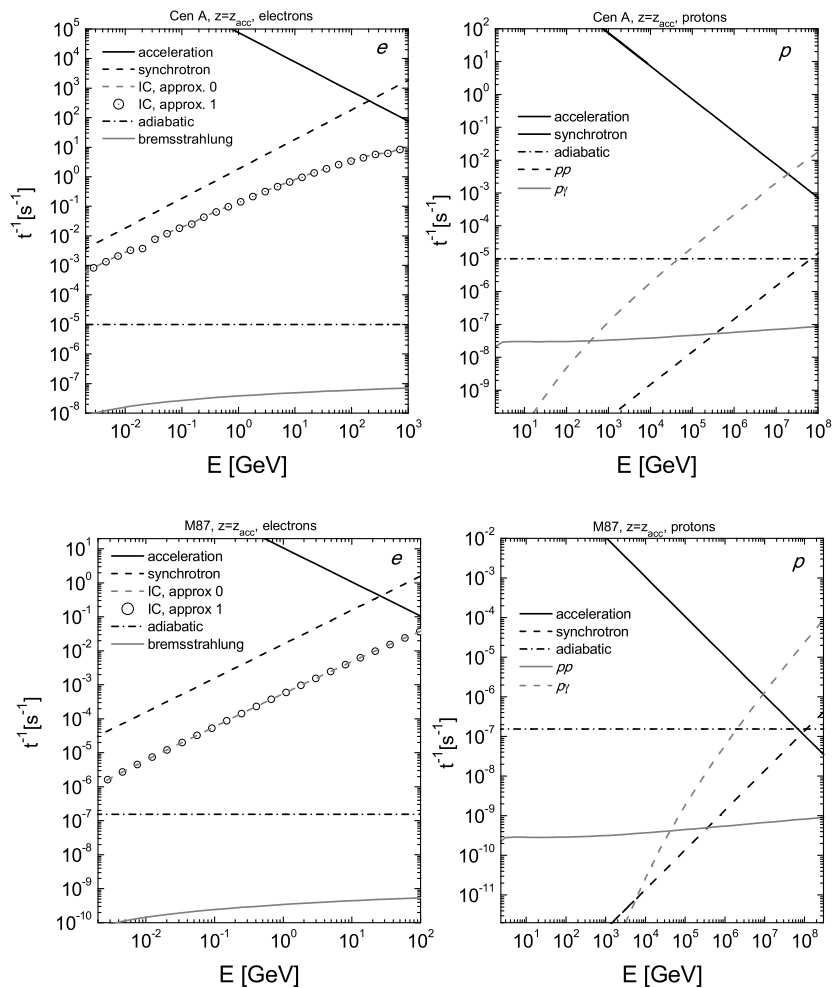

Figure 1: Accelerating and cooling rates for electrons (left) and for protons (right) at a distance $z=z_{\text {acc }}$ from the central engine of Cen A (top) and M87 (bottom) 
target photons corresponding to the synchrotron emission of electrons. From the balance between radiative loss rate and acceleration rate, the maximum energy achievable for protons inside the jet is about $6 \times 10^{17} \mathrm{eV}$ for M87 and Cen A. These protons do not escape from the source. The only possibility to obtain a detectable cosmic rays flux at the earth coming from M87 or Cen A should depend on neutrons produced by pion photoproduction processes and relativistically beamed along the jet direction and Doppler boosted in energy.

The SED of Cen A (see Fig. 2, left ) includes the HESS spectrum in the VHE range [11], the HE energy data from Fermi [21] together with data from CGRO/COMPTEL, RXTE and INTEGRAL. We also include data from $H S T /$ NICMOS and WFPC2, SCUBA at $800 \mu \mathrm{m}$, ISO and SCUBA (450 $\mu \mathrm{m}$ and $850 \mu \mathrm{m}), X M M$-Newton and Chandra spectra. Finally, we also show the old data from the NASA Extragalactic Database. See [21] and references therein for details on these data sets.
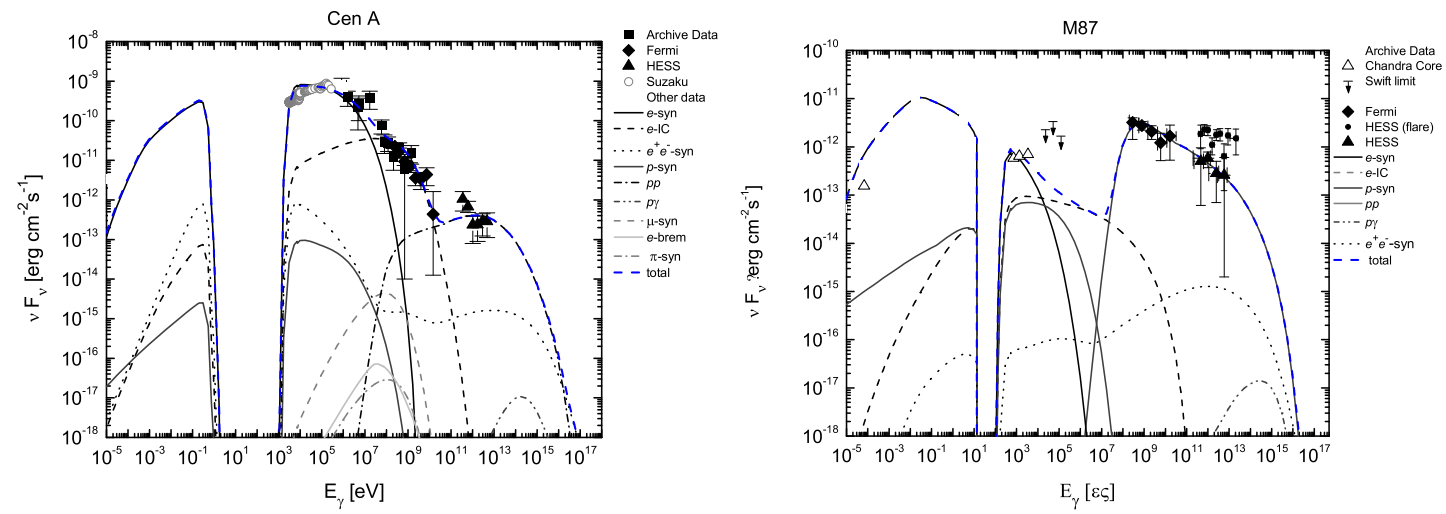

Figure 2: Left: Model output for the SED of Cen A. The different emission processes are indicated, together with the total output. The data points correspond to the references given in the text. Right: Model output for M87 SED taking into account the recent Fermi/LAT, HESS spectra in two different states and historical multi wavelength data.

Since the set of measurements composing the SED of Cen A is quite inhomogeneous in time and angular resolution, so one should be very careful in attempting to interpret any fit of all the spectrum simultaneously. The lack of a good spatial resolution makes impossible to distinguish the emission components (jet, nucleus or other radiation sources). Furthermore, we note a discrepancy between the flux normalization of Fermi/LAT and HESS, which is not yet fully understood.

Nevertheless, we have obtained using our model a spectral energy distribution which is basically consistent with the multi wavelength emission from Cen A which present a mostly steady behavior. The Fermi/LAT analysis revealed that the high-energy spectrum is non-variable over the first year of scientific operation of the instrument. The HESS experiment, also reported a constant flux from Cen A.

In right side of Fig. 2 we plot the contributions to the SED of M87 obtained with the different emitting proceeses in our model. We also show the corresponding multi wavelength observational data. The HESS spectrum included in the VHE range corresponds to a low state from the 2004 observing season [13]. Data from Fermi/LAT [15], 2009 MOJAVE VLBA $15 \mathrm{GHz}$ and Chandra Xray measurements of the core are also shown. We also include the $3 \sigma$ upper limits of the integrated emission in three hard X-ray bands based on the Swift/BAT dataset. Historical measurements of 
the core are also plotted: VLA 1.5, 5, $15 \mathrm{GHz}$, IRAM $89 \mathrm{GHz}$, SMA $230 \mathrm{GHz}$, Spitzer 70, $24 \mu \mathrm{m}$, Gemini $10.8 \mu \mathrm{m}$ and HST optical/UV. See [15] and references therein for details on these data sets.

We have obtained, using our model, a spectral energy distribution which is basically consistent with the multi wavelength emission from M87. The spectrum detected by the Fermi/LAT collaboration connects smoothly with the low-state TeV spectrum detected by HESS. Our model reproduces this connection effectively with the $\gamma$-ray emission produced by $p p$ collisions. Contrary to the case of Cen A, this radiative output for M87 is possible if particles are not so efficiently accelerated $\left(\eta=10^{-4}\right)$ to high energies with a rather steep spectral index $(s=2.4)$.

The accompanying high energy neutrino flux for both sources was calculated using also the set of parameters of Table 1. We show the differential neutrino flux weighted by the squared energy as a function of energy in Fig. 3, where we also plot the estimated sensitivity of KM3Net [22] and IceCube [23] for one year of operation.
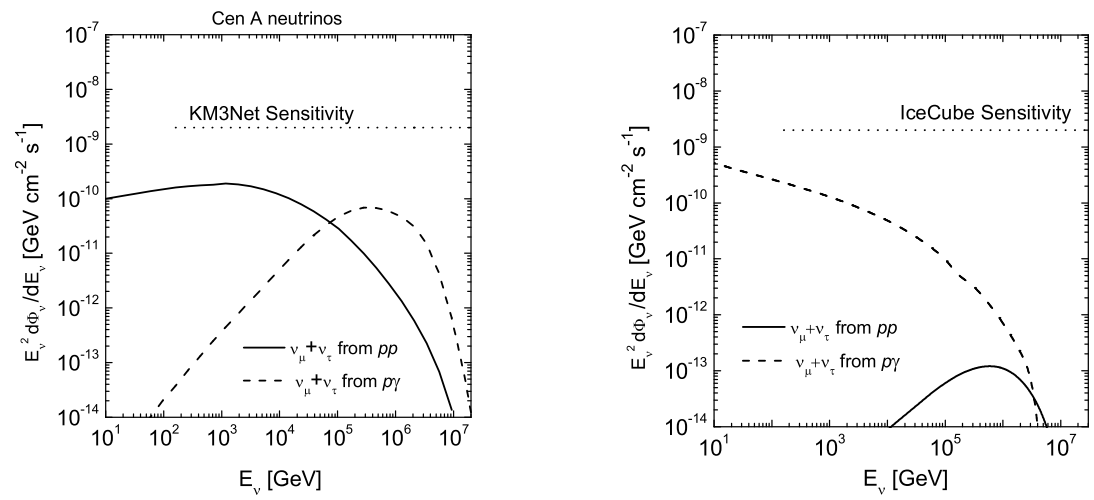

Figure 3: Differential neutrino flux weighted by the squared energy as predicted by the model for Cen A (left) and M87 (right). Dotted line: Approximate KM3Net and IceCube sensitivity for 1 year operation .

\section{Discussion}

We have presented here a lepto-hadronic model describing the particle propagation and interactions in the inclined jets of radiogalaxies. One very important aspect of the present model is that it does not consist of a one-zone treatment, since particles are allowed to convect along the jet, away from the injection zone. The primary electrons are important mainly in the injection zone, since they undergo a rapid synchrotron cooling. Instead, protons are convected away along the jet, loosing energy gradually (mainly by adiabatic cooling, synchrotron radiation, and $p p$ interactions). We have found that the radiative output obtained can account for much of the observational data for the two radiogalaxies M87 and Cen A. As seen in Fig. 2 the model allows for the possibility of explaining the VHE emission by means of $p p$ interactions. The hard X-ray data corresponds to electron synchrotron radiation whereas the soft- $\gamma$ emission is well reproduced by proton synchrotron and IC emission.

A very strong absorption effect is found to modulate the SED for energies $E_{\gamma} \gtrsim 13 \mathrm{eV}$ due to photoionization in the surrounding gas and dust. In the case of Cen A, the large column density 
$N_{H}=10^{23} \mathrm{~cm}^{-2}$ e.g. [24] leads to very important absorption over a wide energy window $E_{\gamma} \lesssim 100$ $\mathrm{eV}$. This is, yet, consistent with observations.

As for the neutrino flux, $p \gamma$ interactions in the acceleration zone of Cen A, would lead to an important neutrino signal, above the corresponding to $p p$ collisions. In the case of M87, the signal is very weak at $E_{v}>1 \mathrm{TeV}$, given the steep spectral index adopted $(s=2.4)$.

For future work, we leave the inclusion of a time derivative term and a time-dependant injection in the transport equation for flaring states.

\section{References}

[1] Böttcher, M. In Astronomical Society of the Pacific Conference Series 373, The Central Engine of Active Galactic Nuclei, ed. L. C. Ho \& J.-W. Wang (2007) 169-+.

[2] Katarzyński, K., Sol, H., \& Kus, A.A\&A 367 (2001) 809.

[3] Lenain, J.-P., Boisson, C., Sol, H., \& Katarzyński, K. A\&A 478 (2008) 111.

[4] Ghisellini, G., Tavecchio, F., \& Chiaberge, M. A\&A 432 (2005) 401.

[5] Reimer, A., Protheroe, R. J., \& Donea, A. A\&A 419 (2004) 89.

[6] Hardcastle, M. J., Cheung, C. C., Feain, I. J., \& Stawarz, Ł. MNRAS 393 (2009) 1041.

[7] Romero, G. E., Combi, J. A., Perez Bergliaffa, S. E., \& Anchordoqui, L. A. Astrop. Physics 5 (1996) 279.

[8] Orellana, M. \& Romero, G. E. In American Institute of Physics Conference Series, 1123, American Institute of Physics Conference Series, ed. C. J. Solano Salinas, J. Bellido, D. Wahl, \& O. Saavedra, 242-243 (2009).

[9] Neronov, A. \& Aharonian, F. A. ApJ 671 (2007) 85.

[10] Rieger, F. M. \& Aharonian, F. A. A\&A 479 (2008) L5

[11] Aharonian, F., Akhperjanian, A. G., Anton, G., et al. ApJ Letters 695 (2009) L40.

[12] Aharonian, F., Akhperjanian, A., Beilicke, M., et al. A\&A 403 (2003) L1.

[13] Aharonian, F., Akhperjanian, A. G., Bazer-Bachi, A. R., et al. Science 314 (2006) 1424.

[14] Abdo, A. A., Ackermann, M., Ajello, M., et al. ApJ 707 (2009) 1310.

[15] Abdo, A. A., Ackermann, M., Ajello, M., et al. ApJ 707 (2009) 55.

[16] McKinney, J. C. MNRAS 368 (2006) 1561.

[17] Komissarov, S. S., Barkov, M. V., Vlahakis, N., \& Königl, A. MNRAS 380 (2007) 51.

[18] Krolik, J. H. Active galactic nuclei : from the central black hole to the galactic environment, ed. Princeton University Press, Princeton (1999).

[19] Ryter, C. E. ApSs, 236 (1996) 285.

[20] Amsler, C., Doser, M., Antonelli, M., et al. Physics Letters B 667 (2008) 1.

[21] Abdo, A. A.,Ackermann, M., Ajello, M. Atwood, W. B., et al. ApJ 719 (2010) 1433-1444.

[22] Katz, U. F., for the KM3NeT Consortium, \& The KM3NeT Consortium.NIM A 602 (2009) 40.

[23] Ahrens, J., Bahcall, J. N., Bai, X., et al. Astrop. Physics 20 (2004) 507.

[24] Morganti, R., Oosterloo, T., Struve, C., Saripalli, L. A\&A 485 (2008) L5. 